PROCEEDINGS OF THE

AMERICAN MATHEMATICAL SOCIETY

Volume 124, Number 12, December 1996, Pages 3729-3737

S 0002-9939(96)03674-X

\title{
OSCILLATION OF FIRST ORDER DELAY DIFFERENTIAL EQUATIONS
}

\author{
BINGTUAN LI
}

(Communicated by Hal L. Smith)

\begin{abstract}
We introduce a new technique to analyze the generalized characteristic equations to obtain some infinite integral conditions for oscillation of the nonautonomous delay differential equations.
\end{abstract}

\section{INTRODUCTION}

Consider the first order delay differential equation

$$
x^{\prime}(t)+p(t) x(t-\tau)=0
$$

where $p(t) \geq 0$ is a continuous function and $\tau$ is a positive constant, or the more general one

$$
x^{\prime}(t)+\sum_{i=1}^{n} p_{i}(t) x\left(t-\tau_{i}\right)=0
$$

where $p_{i}(t) \geq 0$ are continuous and $\tau_{i}$ are positive constants.

By a solution of equation (1) (or (2)) we mean a function $x \in C([\bar{t}-\rho, \infty), \mathbf{R}$ ) for some $\bar{t}$, where $\rho=\tau$ (or $\rho=\max _{1 \leq i \leq n}\left\{\tau_{i}\right\}$ ) satisfies equation (1) (or (2)) for all $t \geq \bar{t}$. As is customary, a solution of equation (1) (or (2)) is said to oscillate if it has arbitrarily large zeros.

Ladas [2] and Koplatadze and Chanturia [3] obtained the well-known oscillation criterion for Eq. (1)

$$
\liminf _{t \rightarrow \infty} \int_{t-\tau}^{t} p(s) d s>\frac{1}{e} .
$$

Ladas and Stavroulakis [4] and Arino and Györi [5] established sufficient conditions for oscillation of Eq. (2) which are in some sense extensions of (3) in the case of several delays. These authors based their techniques on the study of functions of the form $x(t) / x\left(t-\tau_{i}\right)$ or the generalized characteristic equations to obtain finite integral conditions for oscillation of equation (1) or (2). Integral conditions like (3) have been employed by many authors in the study of the oscillatory properties of various functional differential equations. For example, see papers by Grammatikopoulos, Grove and Ladas [7], by Ladas and Qian [11], and by Zhang and Gopalsamy [10].

Received by the editors May 12, 1995.

1991 Mathematics Subject Classification. Primary 34K15; Secondary 34C10.

Key words and phrases. Oscillation, nonoscillation, delay differential equations.

(C)1996 American Mathematical Society 
For other relevant results, the reader is referred to Hunt and Yorke [6], Györi [8], Cheng [12], Kwong [14] and Tramov [1].

In a recent paper [16], this author showed that if

$$
\int_{t-\tau}^{t} p(s) d s \geq \frac{1}{e} \quad \text { for some } t_{0}>0
$$

and

$$
\int_{t_{0}}^{\infty} p(t)\left[\int_{t-\tau}^{t} p(s) d s-\frac{1}{e}\right] d t=\infty,
$$

then every solution of Eq. (1) oscillates. This result improves condition (3).

In this paper, we make use of the generalized characteristic equations and a new technique to examine equations (1) and (2). In section 3, we present an infinite integral condition for oscillation of Eq. (1) which indicates that condition (3) or (4) or even the condition

$$
\liminf _{t \rightarrow \infty} \int_{t-\tau}^{t} p(s) d s>0
$$

is no longer necessary. In section 4 , we establish sufficient conditions for oscillation of Eq. (2).

\section{LEMMAS}

We need the following lemmas for the proofs of our main results.

Lemma 1. If

$$
\limsup _{t \rightarrow \infty} \int_{t}^{t+\tau_{i}} p_{i}(s) d s>0,
$$

for some $i$, and $x(t)$ is an eventually positive solution of Eq. (2), then for the same $i$,

$$
\liminf _{t \rightarrow \infty} \frac{x\left(t-\tau_{i}\right)}{x(t)}<\infty .
$$

Proof. In view of the assumption there exist a constant $d>0$ and a sequence $\left\{t_{k}\right\}$ such that $t_{k} \rightarrow \infty$ as $k \rightarrow \infty$ and

$$
\int_{t_{k}}^{t_{k+\tau_{i}}} p_{i}(s) d s \geq d, \quad k=1,2, \ldots
$$

Then there exists $\xi_{k} \in\left(t_{k}, t_{k}+\tau_{i}\right)$ for every $k$ such that

$$
\int_{t_{k}}^{\xi_{k}} p_{i}(s) d s \geq \frac{d}{2} \quad \text { and } \quad \int_{\xi_{k}}^{t_{k}+\tau_{i}} p_{i}(s) d s \geq \frac{d}{2} .
$$

On the other hand, Eq. (2) implies

$$
x^{\prime}(t)+p_{i}(t) x\left(t-\tau_{i}\right) \leq 0
$$

eventually. By integrating (8) over the intervals $\left[t_{k}, \xi_{k}\right]$ and $\left[\xi_{k}, t_{k}+\tau_{i}\right]$, we find

$$
x\left(\xi_{k}\right)-x\left(t_{k}\right)+\int_{t_{k}}^{\xi_{k}} p_{i}(s) x\left(s-\tau_{i}\right) d s \leq 0
$$


and

$$
x\left(t_{k}+\tau_{i}\right)-x\left(\xi_{k}\right)+\int_{\xi_{k}}^{t_{k}+\tau_{i}} p_{i}(s) x\left(s-\tau_{i}\right) d s \leq 0 .
$$

By omitting the first terms in (9) and (10) and by using the decreasing nature of $x(t)$ and $(7)$, we find

or

$$
-x\left(t_{k}\right)+\frac{d}{2}\left(\xi_{k}-\tau_{i}\right) \leq 0 \quad \text { and } \quad-x\left(\xi_{k}\right)+\frac{d}{2} x\left(t_{k}\right) \leq 0
$$

$$
\frac{x\left(\xi_{k}-\tau_{i}\right)}{x\left(\xi_{k}\right)} \leq\left(\frac{2}{d}\right)^{2}
$$

This completes the proof.

Lemma 2. If Eq. (2) has an eventually positive solution, then

$$
\int_{t}^{t+\tau_{i}} p_{i}(s) d s \leq 1, \quad i=1,2, \ldots, n,
$$

eventually.

Proof. See the proof of Theorem 2.1.3 in [9].

\section{Equations With a Single DELAY}

Our objective in this section is to establish the following result.

Theorem 1. Suppose that $\int_{t}^{t+\tau} p(s) d s>0$ for $t \geq t_{0}$ for some $t_{0}>0$ and

$$
\int_{t_{0}}^{\infty} p(t) \ln \left(e \int_{t}^{t+\tau} p(s) d s\right) d t=\infty .
$$

Then every solution of Eq. (1) oscillates.

Proof. Assume the contrary. Then we may have an eventually positive solution $x(t)$ of Eq. (1). Obviously $x(t)$ is eventually monotonically decreasing. Let $\lambda(t)=$ $-x^{\prime}(t) / x(t)$. Clearly for large $t$, function $\lambda(t)$ is nonnegative and continuous, and $x(t)=x\left(t_{1}\right) \exp \left(-\int_{t_{1}}^{t} \lambda(s) d s\right)$, where $x\left(t_{1}\right)>0$ for some $t_{1} \geq t_{0}$. Furthermore, $\lambda(t)$ satisfies the generalized characteristic equation

$$
\lambda(t)=p(t) \exp \left(\int_{t-\tau}^{t} \lambda(s) d s\right) .
$$

One can easily show that

$$
e^{r x} \geq x+\frac{\ln (e r)}{r} \text { for } r>0
$$

and thus

$$
\begin{aligned}
\lambda(t) & =p(t) \exp \left(A(t) \cdot \frac{1}{A(t)} \int_{t-\tau}^{t} \lambda(s) d s\right) \\
& \geq p(t)\left[\frac{1}{A(t)} \int_{t-\tau}^{t} \lambda(s) d s+\frac{\ln (e A(t))}{A(t)}\right]
\end{aligned}
$$


where $A(t)=\int_{t}^{t+\tau} p(s) d s$. It follows that

$$
\lambda(t) \int_{t}^{t+\tau} p(s)-p(t) \int_{t-\tau}^{t} \lambda(s) d s \geq p(t) \ln \left(e \int_{t}^{t+\tau} p(s) d s\right) .
$$

Then, for $N>T$,

$$
\begin{gathered}
\int_{T}^{N} \lambda(t) \int_{t}^{t+\tau} p(s) d s d t-\int_{T}^{N} p(t) \int_{t-\tau}^{t} \lambda(s) d s d t \\
\geq \int_{T}^{N} p(t) \ln \left(e \int_{t}^{t+\tau} p(s) d s\right) d t
\end{gathered}
$$

By interchanging the order of integration, we find

$$
\begin{aligned}
\int_{T}^{N} p(t) \int_{t-\tau}^{t} \lambda(s) d s d t & \geq \int_{T}^{N-T}\left(\int_{s}^{s+\tau} p(t) \lambda(s) d t\right) d s \\
& =\int_{T}^{N-\tau} \lambda(s) \int_{s}^{s+\tau} p(t) d t d s \\
& =\int_{T}^{N-\tau} \lambda(t) \int_{t}^{t+\tau} p(s) d s d t
\end{aligned}
$$

From this and (16), it follows that

$$
\int_{N-\tau}^{N} \lambda(t) \int_{t}^{t+\tau} p(s) d s d t \geq \int_{T}^{N} p(t) \ln \left(e \int_{t}^{t+\tau} p(s) d s\right) d t .
$$

By Lemma 2, we have

$$
\int_{t}^{t+\tau} p(s) d s \leq 1
$$

eventually. Then by using (18) and (19), we have

or

$$
\int_{N-\tau}^{N} \lambda(t) d t \geq \int_{T}^{N} p(t) \ln \left(e \int_{t}^{t+\tau} p(s) d s\right) d t
$$

In view of (12),

$$
\ln \frac{x(N-\tau)}{x(N)} \geq \int_{T}^{N} p(t) \ln \left(e \int_{t}^{t+\tau} p(s) d s\right) d t
$$

On the other hand, (12) implies that there exists a sequence $\left\{t_{n}\right\}$ with $t_{n} \rightarrow \infty$ as $n \rightarrow \infty$ such that

$$
\int_{t_{n}}^{t_{n}+\tau} p(s) d s \geq \frac{1}{e} \text { for all } n
$$

Hence by Lemma 1, we obtain

$$
\liminf _{t \rightarrow \infty} \frac{x(t-\tau)}{x(t)}<\infty .
$$

This contradicts (21) and completes the proof. 
Theorem 1 substantially improves condition (3). In fact, if (3) holds, then

$$
\int_{t_{0}}^{\infty} p(t) d t=\infty
$$

and there exists $c>0$ such that for large $t$,

$$
\ln \left(e \int_{t}^{t+\tau} p(s) d s\right) \geq c
$$

(22) and (23) imply (12). Condition (12) is an evaluation of $p(t)$ and $\int_{t}^{t+\tau} p(s) d s$ in an infinite interval. Obviously, condition $\int_{t}^{t+\tau} p(s) d s>0$ is necessary for (12).

Example 1. Consider the delay differential equation

$$
x^{\prime}(t)+\exp (k \sin t-1) x(t-1)=0
$$

where $p(t)=\exp (k \sin t-1)$ and $k$ is a positive constant. Clearly,

$$
\liminf _{t \rightarrow \infty} \int_{t-1}^{t} p(s) d s<1 / e .
$$

So condition (3) is not satisfied. By Jensen's inequality,

$$
\begin{aligned}
\int_{0}^{\infty} p(t) \ln \left(e \int_{t}^{t+1} p(s) d s\right) d t & \geq \int_{0}^{\infty} p(t) \int_{t}^{t+1} k \sin s d s d t \\
& =\frac{2 k \sin \frac{1}{2}}{e} \int_{0}^{\infty} \exp (k \sin t) \sin \left(t+\frac{1}{2}\right) d t
\end{aligned}
$$

On the other hand, it is easy to see that $\int_{0}^{t} \exp (k \sin t) \cos t d t$ is bounded and

$$
\int_{0}^{2 \pi} \exp (k \sin t) \sin t d t>0
$$

It follows that $\int_{0}^{\infty} p(t) \ln \left(e \int_{t}^{t+1} p(s) d s\right) d t=\infty$. By Theorem 1, every solution of Eq. (24) oscillates.

We would like to point out that Theorem 1 in [15] is not always true. We use the following example to illustrate this.

Example 2. Consider the delay differential equation

$$
x^{\prime}(t)+\frac{1}{e}\left(1+\frac{1}{t}\right) x(t-1)=0 .
$$

Let $p(t)=\frac{1}{e}\left(1+\frac{1}{t}\right)$. Clearly,

$$
\int_{1}^{\infty} p(t) \ln \left(e \int_{t}^{t+1} p(s) d s\right) d t \geq \frac{1}{e} \int_{1}^{\infty} \ln \left(1+\ln \left(1+\frac{1}{t}\right)\right) d t=\infty .
$$

By Theorem 1, every solution of (25) oscillates. On the other hand, the delay equation

$$
x^{\prime}(t)+\frac{1}{e} x(t-1)=0
$$

has a positive solution, $e^{-t}$. Therefore (25) and (26) possess different oscillatory behaviors though $\lim _{t \rightarrow \infty} \frac{1}{e}\left(1+\frac{1}{t}\right) / \frac{1}{e}=1$.

In the proof of Lemma 2 in [15], the chosen neighborhood $\delta\left(\mu_{0}\right) \subset M$ of $\mu_{0}$ actually depends on the given large constant $m$. As $m \rightarrow+\infty, \delta\left(\mu_{0}\right)$ may not exist. Therefore, the proof is incorrect. 


\section{Equations With SEveral delays}

In this section we obtain sufficient conditions for the oscillation of all solutions of Eq. (2). The main result is the following theorem.

Theorem 2. Let $\tau_{n}=\max \left\{\tau_{1}, \tau_{2}, \ldots, \tau_{n}\right\}$. Suppose that $\sum_{i=1}^{n} \int_{t}^{t+\tau_{i}} p_{i}(s) d s>0$ for $t \geq t_{0}$ for some $t_{0}>0$ and that

$$
\limsup _{t \rightarrow \infty} \int_{t}^{t+\tau_{n}} p_{n}(s) d s>0
$$

If, in addition,

$$
\int_{t_{0}}^{\infty}\left(\sum_{i=1}^{n} p_{i}(t)\right) \ln \left(e \sum_{i=1}^{n} \int_{t}^{t+\tau_{i}} p_{i}(s) d s\right) d t=\infty,
$$

then every solution of Eq. (2) oscillates.

Proof. Assume the contrary. Then Eq. (2) may have an eventually positive and decreasing solution $x(t)$. Let $\lambda(t)=-x^{\prime}(t) / x(t)$. Then $\lambda(t)$ is nonnegative and continuous, and there exists $t_{1} \geq t_{0}$ with $x\left(t_{1}\right)>0$ such that $x(t)=x\left(t_{1}\right) \exp \left(-\int_{t_{1}}^{t} \lambda(s) d s\right)$. Furthermore, $\lambda(t)$ satisfies the generalized characteristic equation

$$
\lambda(t)=\sum_{i=1}^{n} p_{i}(t) \exp \left(\int_{t-\tau_{i}}^{t} \lambda(s) d s\right) .
$$

Let $B(t)=\sum_{i=1}^{n} \int_{t}^{t+\tau_{i}} p_{i}(s) d s$. By using (14), we find

or

$$
\begin{aligned}
\lambda(t) & =\sum_{i=1}^{n} p_{i}(t) \exp \left(B(t) \cdot \frac{1}{B(t)} \int_{t-\tau_{i}}^{t} \lambda(s) d s\right) \\
& \geq \sum_{i=1}^{n} p_{i}(t)\left[\frac{1}{B(t)} \int_{t-\tau_{i}}^{t} \lambda(s) d s+\frac{\ln (e B(t))}{B(t)}\right]
\end{aligned}
$$

$$
\begin{gathered}
\left(\sum_{i=1}^{n} \int_{t}^{t+\tau_{i}} p_{i}(s) d s\right) \lambda(t)-\sum_{i=1}^{n} p_{i}(t) \int_{t-\tau_{i}}^{t} \lambda(s) d s \\
\geq\left(\sum_{i=1}^{n} p_{i}(t)\right) \ln \left(e \sum_{i=1}^{n} \int_{t}^{t+\tau_{i}} p_{i}(s) d s\right) .
\end{gathered}
$$

Then for $N>T$

$$
\begin{gathered}
\int_{T}^{N}\left(\sum_{i=1}^{n} \int_{t}^{t+\tau_{i}} p_{i}(s) d s\right) \lambda(t) d t-\sum_{i=1}^{n} \int_{T}^{N} p_{i}(t) \int_{t-\tau_{i}}^{t} \lambda(s) d s d t \\
\geq \int_{T}^{N}\left(\sum_{i=1}^{n} p_{i}(t)\right) \ln \left(e \sum_{i=1}^{n} \int_{t}^{t+\tau_{i}} p_{i}(s) d s\right) d t .
\end{gathered}
$$

By interchanging the order of integration, we find

$$
\begin{aligned}
\int_{T}^{N} \sum_{i=1}^{n} p_{i}(t) \int_{t-\tau_{i}}^{t} \lambda(s) d s d t & \geq \sum_{i=1}^{n} \int_{T}^{N-\tau_{i}}\left(\int_{s}^{s+\tau_{i}} p_{i}(t) \lambda(s) d t\right) d s \\
& =\sum_{i=1}^{n} \int_{T}^{N-\tau_{i}} \lambda(t) \int_{t}^{t+\tau_{i}} p_{i}(s) d s d t
\end{aligned}
$$


From (30) and (31) it follows that

$$
\sum_{i=1}^{n} \int_{N-\tau_{i}}^{N} \lambda(t) \int_{t}^{t+\tau_{i}} p_{i}(s) d s d t \geq \int_{T}^{N}\left(\sum_{i=1}^{n} p_{i}(t)\right) \ln \left(e \sum_{i=1}^{n} \int_{t}^{t+\tau_{i}} p_{i}(s) d s\right) d t
$$

On the other hand, by Lemma 2, we have

$$
\int_{t}^{t+\tau_{i}} p_{i}(s) d s \leq 1, \quad i=1,2, \ldots, n,
$$

eventually. Then by (32) and (33), we find

$$
\sum_{i=1}^{n} \int_{N-\tau_{i}}^{N} \lambda(t) d t \geq \int_{T}^{N}\left(\sum_{i=1}^{n} p_{i}(t)\right) \ln \left(e \sum_{i=1}^{n} \int_{t}^{t+\tau_{i}} p_{i}(s) d s\right) d t
$$

or

$$
\sum_{i=1}^{n} \ln \frac{x\left(N-\tau_{i}\right)}{x(N)} \geq \int_{T}^{N}\left(\sum_{i=1}^{n} p_{i}(t)\right) \ln \left(e \sum_{i=1}^{n} \int_{t}^{t+\tau_{i}} p_{i}(s) d s\right) d t
$$

In view of $(28)$,

$$
\lim _{t \rightarrow \infty} \prod_{i=1}^{n} \frac{x\left(t-\tau_{i}\right)}{x(t)}=\infty
$$

This implies

$$
\lim _{t \rightarrow \infty} \frac{x\left(t-\tau_{n}\right)}{x(t)}=\infty .
$$

However, by Lemma 1, we have

$$
\liminf _{t \rightarrow \infty} \frac{x\left(t-\tau_{n}\right)}{x(t)}<\infty .
$$

This contradicts (36) and completes the proof.

Corollary 1. If

$$
\liminf _{t \rightarrow \infty} \sum_{i=1}^{n} \int_{t}^{t+\tau_{i}} p_{i}(s) d s>\frac{1}{e},
$$

then every solution of Eq. (2) oscillates.

Proof. Let $\tau_{1}<\tau_{2}<\cdots<\tau_{n}$. Then it follows from (37) that there is an $m$ with $1 \leq m \leq n$ such that

$$
\limsup _{t \rightarrow \infty} \int_{t}^{t+\tau_{m}} p_{m}(s) d s>0
$$

and

$$
\liminf _{t \rightarrow \infty} \sum_{i=1}^{m} \int_{t}^{t+\tau_{i}} p_{i}(s) d s>\frac{1}{e}
$$


Now assume, for the sake of contradiction, that Eq. (2) has an eventually positive solution $x(t)$. Then $x(t)$ is also an eventually positive solution of the inequality

$$
x^{\prime}(t)+\sum_{i=1}^{m} p_{i}(t) x\left(t-\tau_{i}\right) \leq 0 .
$$

So, by Corollary 3.2.2 in [13], we know that the equation

$$
y^{\prime}(t)+\sum_{i=1}^{m} p_{i}(t) y\left(t-\tau_{i}\right)=0
$$

has an eventually positive solution as well. On the other hand, from (39) we see that for some $t_{0}>0$,

$$
\int_{t_{0}}^{\infty}\left(\sum_{i=1}^{m} p_{i}(t)\right) \ln \left(e \sum_{i=1}^{m} \int_{t}^{t+\tau_{i}} p_{i}(s) d s\right) d t=\infty .
$$

Then by Theorem 2, every solution of Eq. (41) oscillates. This is a contradiction and the proof is complete.

\section{ACKNOWLEDGMENT}

I would like to thank Professor Y. Kuang for his advice. Also, I want to thank the referee for making several helpful suggestions.

\section{REFERENCES}

1. M. I. Tramov, Conditions for oscillatory solutions of first order differential equations with a delayed argument, Izvestiya Vysshikh Uchebnykh Zavedenii, Seriya Matematika 1975, no. 3, 92-96; English transl., Soviet Mathematics (Iz. VUZ) 19 (1975). MR 52:961

2. G. Ladas, Sharp conditions for oscillations caused by delays, Applicable Analysis 9 (1979), 93-98. MR 80h:34094

3. R. G. Koplatadze and T. A. Chanturia, On the oscillatory and monotone solutions of the first order differential equations with deviating arguments, Differencial'nye Uravnenija 18 (1982), 1463-1465. (Russian) MR 83k:34069

4. G. Ladas and I. P. Stavroulakis, Oscillations caused by several retarded and advanced arguments, J. Differential Equations 44 (1982), 134-152. MR 83e:34104

5. O. Arino, I. Györi and A. Jawhari, Oscillation criteria in delay equations, J. Differential Equations 53 (1984), 115-123. MR 85k:34168

6. B. R. Hunt and J. A. Yorke, When all solutions of $x^{\prime}=\sum_{j=1}^{n} q_{j}(t) x\left(t-T_{j}(t)\right)$ oscillate, J. Differential Equations 53 (1984), 139-145. MR 85k:34169

7. M. K. Grammatikopoulos, E. A. Grove and G. Ladas, Oscillations of first-order neutral delay differential equations, J. Math. Anal. Appl. 120 (1986), 510-520. MR 87m:34089

8. I. Györi, Oscillation conditions in scalar linear delay differential equations, Bull. Austral. Math. Soc. 34 (1986), 1-9. MR 87j:34137

9. G. S. Ladde, V. Lakshmikantham and B. G. Zhang, Oscillation theory of differential equations with deviating arguments, Marcel Dekker, New York, 1987. MR 90h:34118

10. B. G. Zhang and K. Gopalsamy, Oscillation and nonoscillation in a nonautonomous delaylogistic equation, Quart. Appl. Math. 46 (1988), 267-273. MR 89k:34128

11. G. Ladas and C. Qian, Oscillation in differential equations with positive and negative coeffcients, Canad. Math. Bull. 33 (1990), 442-451. MR 92b:34083

12. Y. Cheng, Oscillation in nonautonomous scalar differential equations with deviating arguments, Proc. Amer. Math. Soc. 110 (1990), 711-719. MR 91b:34110

13. I. Györi and G. Ladas, Oscillation theory of delay differential equations with applications, Clarendon Press, Oxford, 1991. MR 93m:34109

14. M. K. Kwong, Oscillation of first-order delay equations, J. Math. Anal. Appl. 156 (1991), 274-286. MR 92b:34082 
15. G. Ladas, C. Qian and J. Yan, A comparison result for the oscillation of delay differential equations, Proc. Amer. Math. Soc. 114 (1992), 939-946. MR 92g:34097

16. B. Li, Oscillations of delay differential equations with variable coefficients, J. Math. Anal. Appl. 192 (1995), 312-321. CMP 95:12

17. L. H. Erbe, Qingkai Kong and B. G. Zhang, Oscillation theory for functional differential equations, Marcel Dekker, New York, 1995. CMP 95:6

Department of Mathematics, Arizona State University, Tempe, Arizona 85287-1804

E-mail address: bingtuan@asu.edu 